PROCEEDINGS OF THE

AMERICAN MATHEMATICAL SOCIETY

Volume 137, Number 11, November 2009, Pages 3601-3607

S 0002-9939(09)10004-7

Article electronically published on June 5, 2009

\title{
A $2 \times 2$ LATTICE SPACE-TIME CODE OF THE HIGHEST RANK
}

\author{
SHICHUN YANG, BO HE, AND ALAIN TOGBÉ \\ (Communicated by Wen-Ching Winnie Li)
}

\begin{abstract}
For all previous constructions of $2 \times 2$ lattice space-time codes with a positive diversity product, the rank $r(2)$ was such that $r(2) \leq 4$. An example of a $2 \times 2$ lattice space-time code of rank 5 with a positive diversity product was given by Xing and Li. In this paper, we give an example of a $2 \times 2$ lattice space-time code of rank 8 with a positive diversity product. This gives an answer to the open problem set by Xing and Li.
\end{abstract}

\section{INTRODUCTION}

Let $\mathbb{C}, \mathbb{R}, \mathbb{Z}, \mathbb{N}$ be the sets of all complex numbers, real numbers, integers and positive integers respectively. We denote by $\mathcal{M}_{n}(\mathbb{C})$ the set of $n \times n$ matrices over $\mathbb{C}$. A lattice space-time code over $\mathbb{C}$ is a set $\mathcal{A}$ consisting of matrices in $\mathcal{M}_{n}(\mathbb{C})$ such that $\mathcal{A}$ is a free abelian group under the matrix addition. The rank of this group is called the dimension or rank of $\mathcal{A}$. In recent years, the study of space-time codes has generated much interest. Many mathematical areas such as number theory, algebra, combinatorics, etc., have been employed to construct good lattice codes (11, 2], 5], 6], 7], 8], 11, 9], 12, 13, 14]).

The diversity product of $\mathcal{A}$ is defined by (see [14])

$$
\delta(\mathcal{A}):=\inf \{|\operatorname{det}(A-B)|: A, B \in \mathcal{A}, A \neq B\} .
$$

The normalized diversity product of $\mathcal{A}$ in $\mathcal{M}_{n}(\mathbb{C})$ is defined by (see [10, [8], [5])

$$
d_{g}=\frac{(\delta(\mathcal{A}))^{2}}{|\operatorname{det} G| \cdot|\mathcal{L}|^{n / 2}}=\frac{(\delta(\mathcal{A}))^{2}}{\sqrt{|\operatorname{det} g|}},
$$

where $G$ is the corresponding generating matrix of the complex lattice $\mathcal{A}, g$ is the corresponding real generating matrix for $\Lambda_{G}$, and $|\mathcal{L}|$ denotes the absolute value of the determinant of the $2 \times 2$ generating matrix of the two-dimensional real base lattice $\mathcal{L}$.

The criteria for lattice space-time codes are: the rank of $\mathcal{A}$ should be as large as possible, the diversity product of $\mathcal{A}$ should be as large as possible, and the discriminant of $\mathcal{A}$ should be as small as possible. A natural question is: what is the maximal rank of a lattice space-time code $\mathcal{A}$ such that $\delta(\mathcal{A})>0$ ?

We define $r(n)$ by (see [14])

$$
r(n):=\max \left\{\operatorname{rank}(\mathcal{A}): \mathcal{A} \text { is a lattice in } \mathcal{M}_{n}(\mathbb{C}), \delta(\mathcal{A})>0\right\} .
$$

Received by the editors February 17, 2009.

2000 Mathematics Subject Classification. Primary 06B99, 15A15; Secondary 11Z05.

Key words and phrases. Rank, diversity product, lattice.

(C)2009 American Mathematical Society 
Determining the exact value for $r(n)$ seems difficult. One obvious lower bound is $r(n) \geq 2 n$ (see [1, 2], 7], 8, 9]). Recently, Xing and Li [14] gave an upper bound $r(n) \leq 2 n^{2}$.

The case $n=2$ is of a particular interest. However, even in this case, we only know that $4 \leq r(n) \leq 8$. No $2 \times 2$ lattice space-time codes of rank greater than 4 with a positive diversity product had been constructed until in 14, Xing and Li gave an example of a $2 \times 2$ lattice space-time code of rank 5 with a positive diversity product and raised the following question.

Open problem. Is there a $2 \times 2$ lattice space-time code of rank greater than 5 with a positive diversity product?

In this paper, we give an example of a $2 \times 2$ lattice space-time code of rank 8 with a positive diversity product (Section 2). Moreover, we prove that if $\mathcal{A}$ is the lattice generated by $A_{j}(1 \leq j \leq 8)$ and if the set $\left\{A_{j}:(1 \leq j \leq 8)\right\}$ is linearly independent over $\mathbb{R}$, then $\delta(\mathcal{A}) \leq a$, where $a$ is a constant, only dependent on $A_{j}(1 \leq j \leq 8)$. See Section 3 .

\section{A $2 \times 2$ LATTICE SPACE-TIME CODE OF RANK 8}

Theorem 2.1. Consider the eight $2 \times 2$ matrices over $\mathbb{C}$ :

$$
A_{1}=\left(\begin{array}{ll}
1 & 0 \\
0 & 1
\end{array}\right), A_{2}=\left(\begin{array}{cc}
-i & 0 \\
0 & i
\end{array}\right), A_{3}=\left(\begin{array}{cc}
0 & 1 \\
-1 & 0
\end{array}\right), A_{4}=\left(\begin{array}{cc}
0 & -i \\
-i & 0
\end{array}\right)
$$

and

$$
A_{5}=\left(\begin{array}{cc}
\sqrt{q} & 0 \\
0 & -\sqrt{q}
\end{array}\right), A_{6}=\left(\begin{array}{cc}
\sqrt{q} i & 0 \\
0 & \sqrt{q} i
\end{array}\right), A_{7}=\left(\begin{array}{cc}
0 & \sqrt{q} \\
\sqrt{q} & 0
\end{array}\right), A_{8}=\left(\begin{array}{cc}
0 & \sqrt{q} i \\
-\sqrt{q} i & 0
\end{array}\right),
$$

where $q$ is a positive integer such that $q \equiv 7(\bmod 8)$. Let $\mathcal{A}$ be the lattice generated by $A_{1}, A_{2}, A_{3}, A_{4}, A_{5}, A_{6}, A_{7}$ and $A_{8}$. Then the rank of $\mathcal{A}$ is 8 and the diversity product of $\mathcal{A}$ is 1 .

Proof. First of all, it is clear that $\left\{A_{1}, A_{2}, \ldots, A_{8}\right\}$ is linearly independent over $\mathbb{R}$. Let

$$
\begin{aligned}
& A=x_{1} A_{1}+x_{2} A_{2}+x_{3} A_{3}+x_{4} A_{4}+y_{1} A_{5}+y_{2} A_{6}+y_{3} A_{7}+y_{4} A_{8} \\
= & \left(\begin{array}{cc}
x_{1}+y_{1} \sqrt{q}+\left(-x_{2}+y_{2} \sqrt{q}\right) i & x_{3}+y_{3} \sqrt{q}+\left(-x_{4}+y_{4} \sqrt{q}\right) i \\
-x_{3}+y_{3} \sqrt{q}+\left(-x_{4}-y_{4} \sqrt{q}\right) i & x_{1}-y_{1} \sqrt{q}+\left(x_{2}+y_{2} \sqrt{q}\right) i
\end{array}\right) .
\end{aligned}
$$

To show that the rank of $\mathcal{A}$ is 8 and the diversity product of $\mathcal{A}$ is at least 1 , it is sufficient to prove that for any eight integers $x_{1}, x_{2}, x_{3}, x_{4}, y_{1}, y_{2}, y_{3}, y_{4}$ with $\left(x_{1}, x_{2}, x_{3}, x_{4}, y_{1}, y_{2}, y_{3}, y_{4}\right) \neq(0,0,0,0,0,0,0,0)$, we have $|\operatorname{det}(A)| \geq 1$. Without loss of generality, we assume that $\operatorname{gcd}\left(x_{1}, x_{2}, x_{3}, x_{4}, y_{1}, y_{2}, y_{3}, y_{4}\right)=1$.

From (2.1), we get

$$
\begin{aligned}
\operatorname{det}(A)=x_{1}^{2}+x_{2}^{2}+x_{3}^{2}+x_{4}^{2}- & q y_{1}^{2}-q y_{2}^{2}-q y_{3}^{2}-q y_{4}^{2} \\
& +2\left(x_{1} y_{2}+x_{2} y_{1}+x_{3} y_{4}+x_{4} y_{3}\right) \sqrt{q} i
\end{aligned}
$$

and so

$$
\begin{aligned}
|\operatorname{det}(A)|^{2}=\left(x_{1}^{2}+x_{2}^{2}+x_{3}^{2}+x_{4}^{2}-\right. & \left.q y_{1}^{2}-q y_{2}^{2}-q y_{3}^{2}-q y_{4}^{2}\right)^{2} \\
& +4 q\left(x_{1} y_{2}+x_{2} y_{1}+x_{3} y_{4}+x_{4} y_{3}\right)^{2} .
\end{aligned}
$$


If one of the two terms $x_{1}^{2}+x_{2}^{2}+x_{3}^{2}+x_{4}^{2}-q y_{1}^{2}-q y_{2}^{2}-q y_{3}^{2}-q y_{4}^{2}$ and $x_{1} y_{2}+x_{2} y_{1}+$ $x_{3} y_{4}+x_{4} y_{3}$ is not zero, then $|\operatorname{det}(A)| \geq \min \{1,2 \sqrt{q}\}=1$. Therefore we assume that

$$
x_{1}^{2}+x_{2}^{2}+x_{3}^{2}+x_{4}^{2}-q y_{1}^{2}-q y_{2}^{2}-q y_{3}^{2}-q y_{4}^{2}=0
$$

and

$$
x_{1} y_{2}+x_{2} y_{1}+x_{3} y_{4}+x_{4} y_{3}=0 .
$$

Now, equations (2.2) and (2.3) imply that

(2.4) $\left(x_{1}+y_{2}\right)^{2}+\left(x_{2}+y_{1}\right)^{2}+\left(x_{3}+y_{4}\right)^{2}+\left(x_{4}+y_{3}\right)^{2}=(1+q)\left(y_{1}^{2}+y_{2}^{2}+y_{3}^{2}+y_{4}^{2}\right)$.

It follows that

$$
\left(x_{1}+y_{2}\right)^{2}+\left(x_{2}+y_{1}\right)^{2}+\left(x_{3}+y_{4}\right)^{2}+\left(x_{4}+y_{3}\right)^{2} \equiv 0 \quad(\bmod 8) .
$$

Since the square of an integer is always $\equiv 0,1$ or $4(\bmod 8)$, then

$$
2\left|x_{1}+y_{2}, 2\right| x_{2}+y_{1}, 2\left|x_{3}+y_{4}, 2\right| x_{4}+y_{3} \text {. }
$$

So $x_{1}$ and $y_{2}, x_{2}$ and $y_{1}, x_{3}$ and $y_{4}, x_{4}$ and $y_{3}$ are of the same parity. On the other hand, from (2.3), there are three possibilities:

(i) all $x_{1} y_{2}, x_{2} y_{1}, x_{3} y_{4}$, and $x_{4} y_{3}$ are even;

(ii) two of $x_{1} y_{2}, x_{2} y_{1}, x_{3} y_{4}, x_{4} y_{3}$ are odd and the other two are even;

(iii) all $x_{1} y_{2}, x_{2} y_{1}, x_{3} y_{4}$, and $x_{4} y_{3}$ are odd.

$C$ ase (i) We suppose that all $x_{1} y_{2}, x_{2} y_{1}, x_{3} y_{4}$, and $x_{4} y_{3}$ are even. As $2 \mid x_{1}+$ $y_{2}$ and $2 \mid x_{1} y_{2}$, then we have $2 \mid x_{1}$ and $2 \mid y_{2}$. By the same argument, the other 6 variables are all even. Therefore, with $\operatorname{gcd}\left(x_{1}, x_{2}, x_{3}, x_{4}, y_{1}, y_{2}, y_{3}, y_{4}\right)=1$, we obtain a contradiction.

$C$ ase (ii) Now we suppose that two of $x_{1} y_{2}, x_{2} y_{1}, x_{3} y_{4}, x_{4} y_{3}$ are odd and the other two are even. Without loss of generality, we assume

$$
2 \nmid x_{1} y_{2}, 2 \nmid x_{2} y_{1} \text { and } 2\left|x_{3} y_{4}, 2\right| x_{4} y_{3} .
$$

Then $x_{1}, x_{2}, y_{1}, y_{2}$ are odd, and $x_{3}, x_{4}, y_{3}, y_{4}$ are even. From (2.3), we deduce the following congruence:

$$
x_{1} y_{2}+x_{2} y_{1} \equiv-\left(x_{3} y_{4}+x_{4} y_{3}\right) \equiv 0 \quad(\bmod 4) .
$$

This implies that, by considerations modulo 4 , one of $x_{1} y_{2}, x_{2} y_{1}$ is 1 and the other is 3 , say $x_{1} y_{2} \equiv 1(\bmod 4)$ and $x_{2} y_{1} \equiv 3(\bmod 4)$. It follows that $x_{1}+y_{2} \equiv 2$ $(\bmod 4)$ and $x_{2}+y_{1} \equiv 0(\bmod 4)$. Hence, by (2.4) and $2 \mid y_{1}^{2}+y_{2}^{2}+y_{3}^{2}+y_{4}^{2}$ we have

$$
4+\left(x_{3}+y_{4}\right)^{2}+\left(x_{4}+y_{3}\right)^{2} \equiv 0 \quad(\bmod 16) .
$$

This and the fact that $\left(x_{3}+y_{4}\right)^{2},\left(x_{4}+y_{3}\right)^{2} \equiv 0$ or $4(\bmod 16)$ lead to a contradiction.

Case (iii) Finally, we take all $x_{1} y_{2}, x_{2} y_{1}, x_{3} y_{4}$, and $x_{4} y_{3}$ to be odd. Since the $x_{i}$ and $y_{i}$ are all odd, then equation (2.4) gives us

$$
\left(x_{1}+y_{2}\right)^{2}+\left(x_{2}+y_{1}\right)^{2}+\left(x_{3}+y_{4}\right)^{2}+\left(x_{4}+y_{3}\right)^{2} \equiv 0 \quad(\bmod 32) .
$$

So we obtain

$$
\left(\frac{x_{1}+y_{2}}{2}\right)^{2}+\left(\frac{x_{2}+y_{1}}{2}\right)^{2}+\left(\frac{x_{3}+y_{4}}{2}\right)^{2}+\left(\frac{x_{4}+y_{3}}{2}\right)^{2} \equiv 0 \quad(\bmod 8) .
$$

This implies that $\frac{x_{1}+y_{2}}{2}, \frac{x_{2}+y_{1}}{2}, \frac{x_{3}+y_{4}}{2}$ and $\frac{x_{4}+y_{3}}{2}$ are both even. 
On the other hand, from equations (2.2) and (2.3) we have

$$
\left(x_{1}-y_{2}\right)^{2}+\left(x_{2}-y_{1}\right)^{2}+\left(x_{3}-y_{4}\right)^{2}+\left(x_{4}-y_{3}\right)^{2}=(q+1)\left(y_{1}^{2}+y_{2}^{2}+y_{3}^{2}+y_{4}^{2}\right) .
$$

It follows that

$$
\left(\frac{x_{1}-y_{2}}{2}\right)^{2}+\left(\frac{x_{2}-y_{1}}{2}\right)^{2}+\left(\frac{x_{3}-y_{4}}{2}\right)^{2}+\left(\frac{x_{4}-y_{3}}{2}\right)^{2} \equiv 0 \quad(\bmod 8) .
$$

Therefore, $\frac{x_{1}-y_{2}}{2}, \frac{x_{2}-y_{1}}{2}, \frac{x_{3}-y_{4}}{2}$, and $\frac{x_{4}-y_{3}}{2}$ are also even. Thus we have

$$
2 \mid \frac{x_{1}+y_{2}}{2}+\frac{x_{1}-y_{2}}{2}=x_{1} .
$$

But $x_{1}$ is an odd integer. This is impossible.

Combining the above three cases, we have shown that equations (2.2) and (2.3) cannot simultaneously hold. Thus we have $|\operatorname{det}(A)| \geq 1$. This completes the proof.

We have the following remarks.

Remark 2.2. (1) The condition $q \equiv 7(\bmod 8)$ can be replaced by $q=2^{2 r}(8 t+$ 7 ), for nonnegative integers $r$ and $t$. To see this, if instead of $q=8 t+7$ we consider $2^{2 r}(8 t+7)$, then we only need to take $2^{r} y_{j}(j=1,2,3,4)$ instead of $y_{j}$ in our proof.

On the other hand, if $q$ doesn't have the form $2^{2 r}(8 t+7)$, then Theorem 2.1 is not true. In this case, we set $x_{1}=x_{2}=x_{3}=y_{3}=0$ and $x_{4}=q$. Thus equation (2.3) is satisfied and equation (2.2) becomes $q^{2}=q\left(y_{1}^{2}+y_{2}^{2}+y_{4}^{2}\right)$, and so we obtain

$$
q=y_{1}^{2}+y_{2}^{2}+y_{4}^{2} .
$$

The above equation has integer solutions $\left(y_{1}, y_{2}, y_{4}\right)$. One can see $[3$, page 271 , or 4, page 133 .

(2) From [10, 5] and using equation (1.2), we can compute the discriminant and the normalized diversity product of the lattice $\mathcal{A}$ given by Theorem 1 .

Letting $z_{1}=x_{1}+y_{2} \sqrt{q} i, z_{2}=x_{2}+y_{1} \sqrt{q} i, z_{3}=x_{3}+y_{4} \sqrt{q} i, z_{4}=$ $x_{4}-y_{3} \sqrt{q} i, z_{1}, z_{2}, z_{3}, z_{4} \in \mathbb{Z}[\sqrt{q} i]$, from (2.1), we get

$$
A=\left(\begin{array}{cc}
z_{1}-i z_{2} & z_{3}-i z_{4} \\
-z_{3}-i z_{4} & z_{1}+i z_{2}
\end{array}\right) .
$$

The generating matrix $G$ of the lattice $\mathcal{A}$ is $G=\left(\begin{array}{cc}G_{1} & 0 \\ 0 & -G_{2}\end{array}\right)$, where $G_{1}=\left(\begin{array}{cc}1 & -i \\ 1 & i\end{array}\right), G_{2}=\left(\begin{array}{cc}1 & -i \\ -1 & -i\end{array}\right)$, and $|\mathcal{L}|=\sqrt{q}$. Then the discriminant of $\mathcal{A}$ is $d_{\mathcal{A}}=-4$, and the normalized diversity product of $\mathcal{A}$ is $d_{g}=\frac{1}{4 \sqrt{q}}$.

\section{An upper bound of Any $2 \times 2$ LATtice SPACE-Time COde}

In this section, we will use a result in Diophantine approximation to get a relative upper bound of $\delta(\mathcal{A})$. 
Lemma 3.1. Let $X \in \mathbb{N}, m, n \in \mathbb{N}$, and $n>m \geq 1$. Let $L_{i}(\vec{x})=\sum_{j=1}^{n} a_{i j} x_{j}$, where $\vec{x}=\left(x_{1}, \cdots, x_{n}\right), a_{i j} \in \mathbb{R}, 1 \leq i \leq m, 1 \leq j \leq n$. If

$$
\sum_{j=1}^{n}\left|a_{i j}\right| \leq A_{i}, i=1, \cdots, m
$$

then there exists a nonvanishing vector $\vec{x}^{(0)}=\left(x_{01}, \cdots, x_{0 n}\right) \in \mathbb{Z}^{n}$, satisfying

$$
\left|L_{i}\left(\vec{x}^{(0)}\right)\right|<A_{i} X^{1-\frac{n}{m}}, i=1, \cdots, m,
$$

and

$$
\left|x_{0 j}\right| \leq X, j=1, \cdots, n \text {. }
$$

Proof. See page 62 of 15.

Now we prove the following theorem.

Theorem 3.2. For any eight $2 \times 2$ matrices over $\mathbb{C}$ :

$$
A_{j}=\left(\begin{array}{ll}
a_{j 1}+a_{j 2} i & a_{j 3}+a_{j 4} i \\
a_{j 5}+a_{j 6} i & a_{j 7}+a_{j 8} i
\end{array}\right)
$$

where $a_{j 1}, a_{j 2}, a_{j 3}, a_{j 4}, a_{j 5}, a_{j 6}, a_{j 7}, a_{j 8} \in \mathbb{R}$, and $\left\{A_{j}(1 \leq j \leq 8)\right\}$ is linearly independent over $\mathbb{R}$. Let $\mathcal{A}$ be the lattice generated by $A_{1}, A_{2}, A_{3}, A_{4}, A_{5}, A_{6}, A_{7}$ and $A_{8}$. Then

$$
\delta(\mathcal{A}) \leq \sqrt{\left(a_{1}^{2}+a_{2}^{2}\right)\left(a_{7}^{2}+a_{8}^{2}\right)}+\sqrt{\left(a_{3}^{2}+a_{4}^{2}\right)\left(a_{5}^{2}+a_{6}^{2}\right)},
$$

where $a_{k}=\sum_{j=1}^{8}\left|a_{j_{k}}\right|, 1 \leq k \leq 8$.

Proof. We assume that $x_{j} \in \mathbb{Z}(1 \leq j \leq 8)$ and let $A=\sum_{j=1}^{8} x_{j} A_{j}$. Then

$$
A=\left(\begin{array}{ll}
\sum_{j=1}^{8} x_{j} a_{j 1}+i \sum_{j=1}^{8} x_{j} a_{j 2} & \sum_{j=1}^{8} x_{j} a_{j 3}+i \sum_{j=1}^{8} x_{j} a_{j 4} \\
\sum_{j=1}^{8} x_{j} a_{j 5}+i \sum_{j=1}^{8} x_{j} a_{j 6} & \sum_{j=1}^{8} x_{j} a_{j 7}+i \sum_{j=1}^{8} x_{j} a_{j 8}
\end{array}\right) .
$$

From Lemma 3.1 there exists nonvanishing vector $\vec{x}^{(0)}=\left(x_{01}, \cdots, x_{08}\right) \in \mathbb{Z}^{8}$, satisfying

$$
\left|\sum_{j=1}^{8} x_{0 j} a_{j k}\right|<a_{k} X^{1-\frac{8}{4}}=\frac{a_{k}}{X}, k=1, \cdots, 4,
$$

and

$$
\left|x_{0 j}\right| \leq X, j=1, \cdots, 8,
$$

where $a_{k}=\sum_{j=1}^{n}\left|a_{j k}\right|, X \in \mathbb{N}$. Thus from (3.1),

$$
\begin{gathered}
\delta(A) \leq\left|\operatorname{det}\left(\begin{array}{cc}
\frac{1}{X}\left(a_{1}+i a_{2}\right) & \frac{1}{X}\left(a_{3}+i a_{4}\right) \\
-\left(a_{5}+i a_{6}\right) X & \left(a_{7}+i a_{8}\right) X
\end{array}\right)\right|=\left|\operatorname{det}\left(\begin{array}{cc}
a_{1}+i a_{2} & a_{3}+i a_{4} \\
-\left(a_{5}+i a_{6}\right) & a_{7}+i a_{8}
\end{array}\right)\right| \\
\leq \sqrt{\left(a_{1}^{2}+a_{2}^{2}\right)\left(a_{7}^{2}+a_{8}^{2}\right)}+\sqrt{\left(a_{3}^{2}+a_{4}^{2}\right)\left(a_{5}^{2}+a_{6}^{2}\right)} .
\end{gathered}
$$

This completes the proof of Theorem 3.2

Remark 3.3. (1) From Theorem 3.2 and the work done in [10, [5], we have

$$
d_{g} \leq \frac{\left(\sqrt{\left(a_{1}^{2}+a_{2}^{2}\right)\left(a_{7}^{2}+a_{8}^{2}\right)}+\sqrt{\left(a_{3}^{2}+a_{4}^{2}\right)\left(a_{5}^{2}+a_{6}^{2}\right)}\right)^{2}}{\sqrt{|\operatorname{det} g|}}
$$

where $g$ is the corresponding real generating matrix for $\Lambda_{G}$. 
(2) Recently, Liao, Wang, and Xia ([5]) constructed a full-rate space-time code based on the optimal quadratic extension on $\mathbb{Q}[i]$ as

$$
C_{2,2}=\left\{\left(\begin{array}{cc}
z_{1}+\exp \left(\frac{i \pi}{6}\right) z_{2} & \rho\left(z_{3}+\exp \left(\frac{i \pi}{6}\right) z_{4}\right) \\
\rho\left(z_{3}+\exp \left(\frac{i 5 \pi}{6}\right) z_{4}\right) & z_{1}+\exp \left(\frac{i 5 \pi}{6}\right) z_{2}
\end{array}\right)\right\}
$$

where $z_{1}, z_{2}, z_{3}, z_{4} \in \mathbb{Z}[i]$ and $\rho=\sqrt{1+i}$. They also constructed a full-rate space-time code based on the optimal quadratic extension on $\mathbb{Q}\left[\zeta_{6}\right]$ as

$$
C_{2,3}=\left\{\left(\begin{array}{cc}
z_{1}+\theta_{1} z_{2} & \rho\left(z_{3}+\theta_{1} z_{4}\right) \\
\rho\left(z_{3}+\theta_{2} z_{4}\right) & z_{1}+\theta_{2} z_{2}
\end{array}\right)\right\}
$$

where $\zeta_{6}=\exp \left(\frac{2 \pi i}{6}\right), z_{1}, z_{2}, z_{3}, z_{4} \in \mathbb{Z}\left[\zeta_{6}\right], \theta_{1}, \theta_{1}$ are the roots of $x^{2}+x+$ $\zeta_{6}$, and $\rho=\sqrt{1+\zeta_{6}}$. The normalized diversity product for code $C_{2,2}$ is $d_{g}=\frac{1}{3 \sqrt{2}} \approx 0.2357$ and the normalized diversity product for code $C_{2,3}$ is $d_{g}=\frac{1}{\frac{3}{4} \sqrt{39}} \approx 0.2135$. These normalized diversity products are both larger than ours obtained in Remark 2.2 (2). In fact, Theorem 3.2 gives a relative upper bound of $d_{g}$.

\section{Acknowledgements}

The authors thank Prof. Xiang-Gen Xia, Department of Electrical and Computer Engineering, University of Delaware, for pointing out to them his papers that help to define the normalized diversity product. They are also grateful to the anonymous referee for insightful and valuable comments that helped to improve the manuscript. The first and second authors were supported by the Natural Science Foundation of the Science and Technology Department of Sichuan Province and the Education Department of Sichuan Province. The third author was supported in part by Purdue University North Central.

\section{REFERENCES}

1. M. O. Damen, A. Tewfik and J. C. Belflore, A construction of a space-time code based on number theory, IEEE Trans. Inform. Theory, Vol. 48, No. 3 (2002), 753-760. MR1889980 (2003i:94003)

2. P. Dayal and M. K. Varanasi, An algebraic family of complex lattices for fading channels with application to space-time codes, IEEE Trans. Inform. Theory, Vol. 51, No. 12 (2005), 4184-4202. MR2243151 (2007b:94153)

3. L. E. Dickson, History of the Theory of Numbers, Volume II: Diophantine Analysis, Dover, New York, 2005.

4. W. J. LeVeque, Topics in Number Theory, Volumes I and II, Dover, New York, 2002. MR 1942365(2003j:11002)

5. H. Liao, H. Wang, and X.-G. Xia, Some designs and normalized diversity product upper bounds for lattice-based diagonal and full-rate space-time block codes, IEEE Trans. Inform. Theory, Vol. 55, No. 2 (2009), 569-583.

6. A. Shokrollahi, B. Hassibi, B. M. Hochwald and W. Sweldens, Representation theory for high-rate multiple-antenna code design, IEEE Trans. Inform. Theory, Vol. 47, No. 6 (2001), 2335-2367. MR1873925 (2003j:94120)

7. V. Tarokh, H. Jafarkhani, and A. R. Calderbank, Space-time block codes from orthogonal designs, IEEE Trans. Inform. Theory, Vol. 45, No. 5 (1999), 1456-1467. MR1699070 (2000g:94001)

8. G. Wang, H. Liao, H. Wang, and X.-G. Xia, Systematic and optimal cyclotomic lattices and diagonal space-time block code designs, IEEE Trans. Inform. Theory, Vol. 50, No. 12 (2004), 3348-3360. MR2103509

9. G. Wang and X.-G. Xia, On optimal multilayer space-time code designs, IEEE Trans. Inform. Theory, Vol. 51, No. 3 (2005), 1102-1135. MR2237973 (2007h:94088a) 
10. G. Wang and X.-G. Xia, Correction to the definition of diversity product in "On optimal multi-layer cyclotomic space-time code designs", IEEE Trans. Inform. Theory, Vol. 51, No. 7 (2005), 2732-2733. MR2246395 (2007h:94088b)

11. H. Wang, G. Wang, and X.-G. Xia, Some $2 \times 2$ unitary space-time codes from sphere packing theory with optimal diversity product of code size 6, IEEE Trans. Inform. Theory, Vol. 50, No. 12 (2004), 3361-3368. MR2103510

12. H. Wang and X.-G. Xia, Optimal normalized diversity product of $2 \times 2$ lattice-based diagonal space-time codes from QAM signal constellations, IEEE Trans. Inform. Theory, Vol. 54, No. 12 (2008), 1814-1818. MR2450310

13. C. P. Xing, Diagonal lattice space-time codes from number fields and asymptotic bounds, IEEE Trans. Inform. Theory, Vol. 53, No. 11 (2007), 3921-3926. MR2446545

14. C. P. Xing and W. Li, A $2 \times 2$ lattice space-time code of rank 5, Proc. Amer. Math. Soc., Vol. 136, No. 10 (2008), 3415-3418. MR2415024

15. Y. C. Zhu and L. X. Wang, An Introduction to Diophantine Approximation, Beijing: Science Press, 1993, 62-63 (in Chinese).

Department of Mathematics, Aba Teachers' College, Wenchuan, Sichuan, 623000, People's Republic of China

E-mail address: ysc1020@sina.com

Department of Mathematics, Aba Teachers' College, Wenchuan, Sichuan, 623000, People's Republic of China

E-mail address: bhe@live.cn

Department of Mathematics, Purdue University North Central, 1401 S, U.S. 421, WeSTVILle, Indiana 46391

E-mail address: atogbe@pnc.edu 